OPEN ACCESS

Edited by: Jianqing Zhao,

Soochow University, China

Reviewed by:

Yong Min Lee,

Daegu Gyeongbuk Institute of Science and Technology (DGIST), South Korea

Zhiqiang Xie,

University of Tennessee Space

Institute (UTSI), United States

*Correspondence:

Sung-Soo Kim

kimss@cnu.ac.kr

tThese authors have contributed equally to this work

Specialty section:

This article was submitted to

Electrochemistry,

a section of the journal

Frontiers in Chemistry

Received: 18 February 2019 Accepted: 14 May 2019

Published: 31 May 2019

Citation:

Lee M, Yoon D, Lee UJ, Umirov N, Mukanova A, Bakenov Z and Kim S-S

(2019) The Electrochemical

Performances of $n$-Type Extended Lattice Spaced Si Negative Electrodes

for Lithium-Ion Batteries.

Front. Chem. 7:389.

doi: 10.3389/fchem.2019.00389

\section{The Electrochemical Performances of $n$-Type Extended Lattice Spaced Si Negative Electrodes for Lithium-Ion Batteries}

\author{
Moonsang Lee ${ }^{1 t}$, Dockyoung Yoon ${ }^{2,3 \dagger}$, Uk Jae Lee ${ }^{4}$, Nurzhan Umirov ${ }^{3}$, Aliya Mukanova ${ }^{5}$, \\ Zhumabay Bakenov ${ }^{5}$ and Sung-Soo $\mathrm{Kim}^{3 *}$ \\ ${ }^{1}$ Korea Basic Science Institute, Daejeon, South Korea, ${ }^{2}$ SK Innovation, Daejeon, South Korea, ${ }^{3}$ Graduate School of Energy \\ Science and Technology, Chungnam National University, Daejeon, South Korea, ${ }^{4}$ School of Integrative Engineering, \\ Chung-Ang University, Seoul, South Korea, ${ }^{5}$ National Laboratory Astana, School of Engineering, Nazarbayev University, \\ Institute of Batteries, Astana, Kazakhstan
}

The electrochemical performances of lithium-ion batteries with different lattice-spacing Si negative electrodes were investigated. To achieve a homogeneous distribution of impurities in the Si anodes, single crystalline Si wafers with As-dopant were ball-milled to form irregular and agglomerated micro-flakes with an average size of $\sim 10 \mu \mathrm{m}$. The structural analysis proved that the As-doped Si negative materials retain the increased lattice constant, thus, keep the existence of the residual tensile stress of around 1.7 GPa compared with undoped Si anode. Electrochemical characterization showed that the As-doped Si anodes have lower discharge capacity, but Coulombic efficiency and capacity retention were improved in contrast with those of the undoped one. This improvement of electrochemical characteristics was attributed to the increased potential barrier on the side of Si anodes, inherited from the electronic and mechanical nature of Si materials doped with As. We believe that this study will guide us the way to optimize the electrochemical performances of LIBs with Si-based anodes.

Keywords: lithium-ion batteries, silicon, dopant, arsenic, discharge capacity, retention

\section{INTRODUCTION}

Lithium-ion batteries (LIBs) have been considered as an important energy storage device for modern portable electronics and electric vehicles (Goodenough and Park, 2013; Nitta et al., 2015; Kim et al., 2016). Until now, the graphite is typically used as anode material in commercial LIBs. However, its theoretical capacity $\left(372 \mathrm{mAh} \mathrm{g}^{-1}\right)$ is not sufficient for the next-generation mobile applications requiring high energy density (Peng et al., 2010; Ma et al., 2014; Ko et al., 2015). To overcome the energy density problem of current LIBs, the use of Si as the anode in LIBs can be an alternative way due to its unique physical properties such as high theoretical capacity of 3,580 mAh $\mathrm{g}^{-1}\left(\mathrm{Li}_{15} \mathrm{Si}_{4}\right.$ state) and low discharge potential ( $<0.5 \mathrm{~V}$ vs. Li/Li $\left.{ }^{+}\right)$(Wang and Dahn, 2006; Liu et al., 2012; Shi et al., 2016). Even if the Si material has such good advantages for LIBs, the commercial success of LIBs with $\mathrm{Si}$ anodes has been impeded by fast capacity fading of Si anodes. This fading results from large volume change more than $300 \%$ of Si anodes during lithiation and delithiation, leading to their disintegration (Ryu et al., 2004; Kim et al., 2011). Furthermore, semiconductor characteristics of Si make it even more difficult to be utilized in the practical application of LIBs as 
a negative electrode owing to the electrical losses due to large ohmic resistance (Wen and Tian, 2013; Wang et al., 2015).

Recently, various research groups have reported that the electrochemical performances of LIBs with $\mathrm{Si}$ anodes can be improved by using Si/Metal alloy electrode (Umirov et al., 2019a,b), the formation of nanostructured-anodes (Mukanova et al., 2018a,b), hollow and yolk-shell structured-composites (Yao et al., 2011; Wang et al., 2013), and doping of Si with impurities such as copper $(\mathrm{Cu})$ (Wen and Tian, 2013), arsenic (As) (McSweeney et al., 2014), aluminum (Al) (Legrain and Manzhos, 2015), silver (Ag) (Talla et al., 2015), boron (B) (Long et al., 2011; Rousselot et al., 2012; Yi et al., 2013), nitrogen (N) (Han et al., 2016), and phosphorus (P) (Domi et al., 2016). Among other approaches, incorporating dopants into Si architectures has gained considerable attention as a suitable route for improving the rate capability, electrical conductivity, and capacity retention of the Si anodes (Mukanova et al., 2018b).

To date, many methods have been proposed for the Si powder preparation, including solution and solid-state based techniques, etc. Taking into account that the electronic/silicon-based semiconductor technologies have widely entered into society and industry, it is easy to imagine how much semiconductor class $\mathrm{Si}$ containing waste is produced every day. These silicon materials can be recycled for use in LIBs.

In this paper, we used the Si wafer in order to demonstrate the possibility of the Si electronic wastes to be reused in LIBs. As-modulated Si micro-flake anodes were investigated as an anode. To achieve a homogeneous distribution of As-impurity in the Si anodes, As-doped Si wafer was prepared by ballmilling and employed for the anode in a lithium cell. Moreover, this work covers the comparative analysis in the structural and electrochemical responses of As-doped and undoped Si microflake anodes to investigate the effects of the lattice spacing in $\mathrm{Si}$ anodes.

We believe that this paper will shed lights on the trends in the electrochemical performance [cycle retention, Coulombic efficiency (CE), and rate capability] of LIBs with the Si microflake anode with different lattice spacing.

\section{EXPERIMENTAL}

The commercial As-doped single crystalline (100) Si wafers with a doping concentration of $1 \times 10^{19} \mathrm{~cm}^{-3}$ were provided by SK Hynix and used as the anode material for LIBs. The doping concentrations of Si wafer were confirmed by secondary ion mass spectrometry (SIMS) (not shown in this paper). Furthermore, the intrinsic (undoped) Si wafers were used as the control samples to compare the effects of As doping. To remove native oxide on Si surface, Si wafers were immersed in diluted $10 \%$ hydrofluoric acid solution for $3 \mathrm{~min}$ and followed by rinsing in deionized (DI) water of ultra-purity (resistance $18.2 \mathrm{M} \Omega \mathrm{cm}$ ) for $5 \mathrm{~min}$. Si wafers were mechanically grounded to achieve Si microflakes using zirconia balls for $2 \mathrm{~h}$ in paint shaker (KM-2000T, Nara Sci).

The undoped and As-doped $\mathrm{Si}$ based electrodes were fabricated via direct casting of slurry onto $\mathrm{Cu}$ foil using the Dr. Blade method. The slurry was prepared by thoroughly
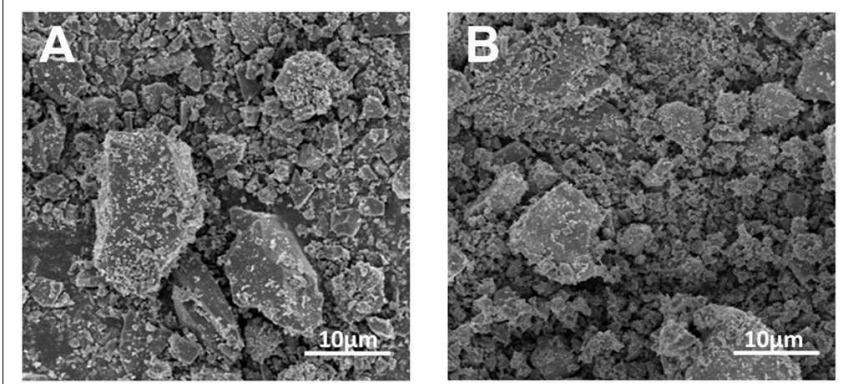

FIGURE 1 | Plan-view SEM image of (A) undoped and (B) As-doped Si powders after ball-milling.

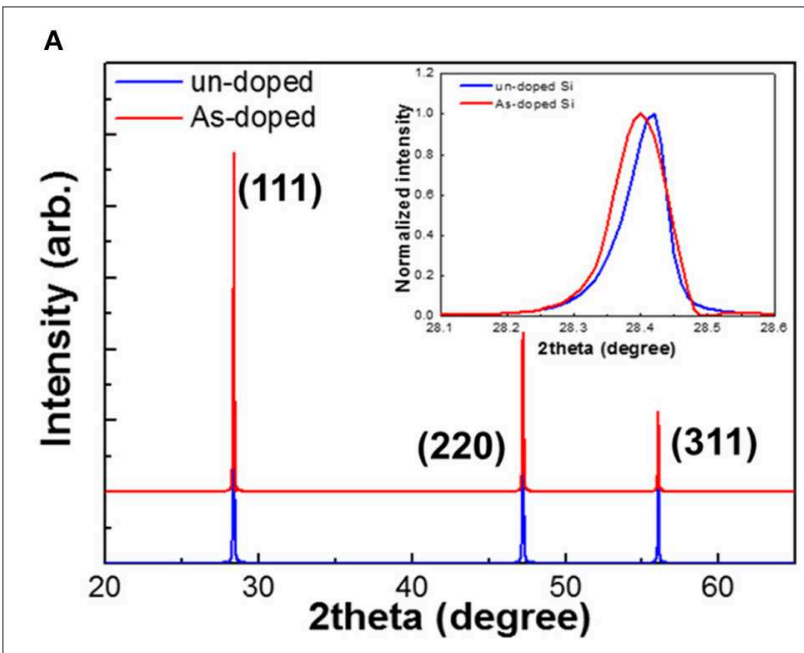

B

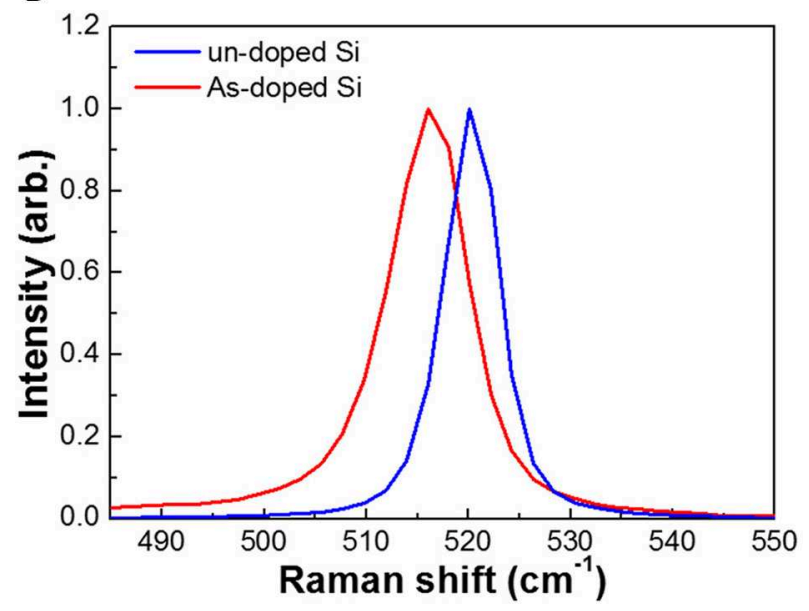

FIGURE 2 | (A) XRD patterns and (B) Raman spectra of undoped and As-doped Si anodes.

mixing of active material and PAI (Polyamide-Imide, Solvay Torlon 4000T) binder with the wt.\% ratio of 9:1, respectively, in NMP (N-Methyl-2-pyrrolidone, Merck) solvent. After casting the electrodes were dried in convection oven at $110^{\circ} \mathrm{C}$ to remove NMP. The loading level of electrodes were adjusted to $5 \mathrm{mg} / \mathrm{cm}^{2}$. 


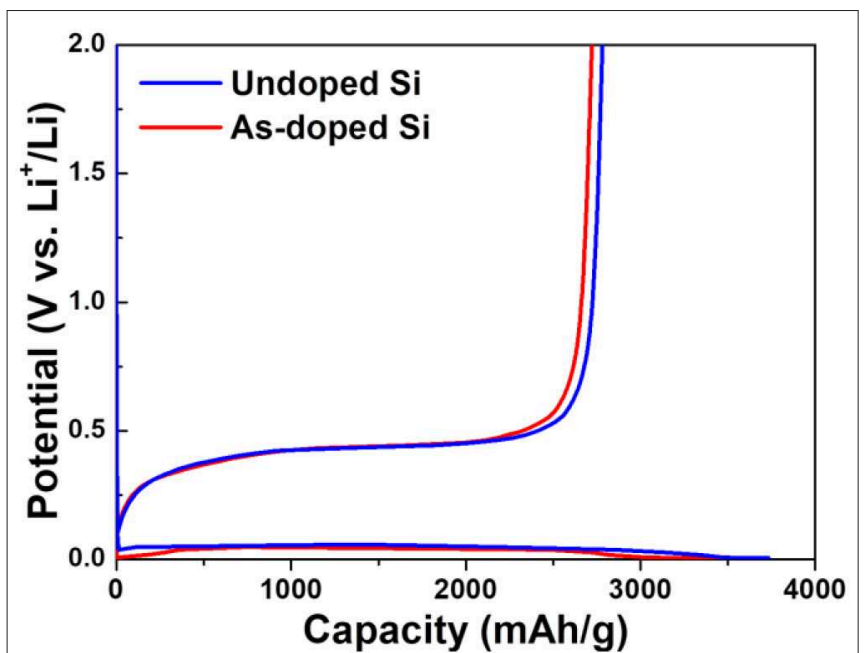

FIGURE 3 | Typical galvanostatic first charge-discharge curves of undoped and As-doped Si electrodes in the potential range from 0.005 to $2.000 \mathrm{~V}$ vs. $\mathrm{Li} / \mathrm{Li}^{+}$at $1.0 \mathrm{~A} \mathrm{~g}^{-1}$.

Electrochemical performances were studied using coin-type half configuration cells with Li metal as the counter electrodes, which were assembled in an Ar-filled glove box with $<1 \mathrm{ppm}$ of both oxygen and moisture. The electrolyte solution used was $1.3 \mathrm{M}$ lithium hexafluoro phosphate $\left(\mathrm{LiPF}_{6}\right)$ dissolved in a solvent mixture of ethylene carbonate (EC) and diethyl carbonate (DEC) with a 3:7 volume ratio. Galvanostatic measurements were carried out to determine charge-discharge capacity and cycling retention in the potential range between 0.005 and $2.000 \mathrm{~V}$ vs. $\mathrm{Li} / \mathrm{Li}^{+}$under a current density of $1.0 \mathrm{~A} \mathrm{~g}^{-1}(0.28 \mathrm{C})$ using a computer-controlled battery measurement system (TOSCAT $3000 \mathrm{U})$.

The surface morphologies of $\mathrm{Si}$ microflake anodes were evaluated by field emission scanning electron microscope (FESEM). The stress evolution, phase transition, and crystallinity of $\mathrm{Si}$ microflake anodes were investigated by micro Raman spectroscopy and X-ray diffraction (XRD).

\section{RESULTS AND DISCUSSION}

Figures 1A,B shows the plan-view SEM images of undoped and As-doped Si negative electrodes, respectively, after ball-milling for $2 \mathrm{~h}$. The irregular and agglomerated particles were made up with the average size of $10 \mu \mathrm{m}$ for both undoped and As-doped $\mathrm{Si}$ anodes. The particle size and distributions were measured by particle size analyzer (not shown in this paper). The similar morphology and size of micro particles of undoped and Asdoped Si anodes were observed, indicating that As-dopants do not influence the morphology and particle size distribution of negative anodes.

Figure 2 exhibits XRD patterns and micro-Raman spectra of undoped and As-doped Si negative electrodes. The phase and crystallinity of $\mathrm{Si}$ anodes used in this work were investigated by XRD as shown in Figure 2A. It should be noted that all XRD peaks are sharp. This feature could be ascribed to the property of
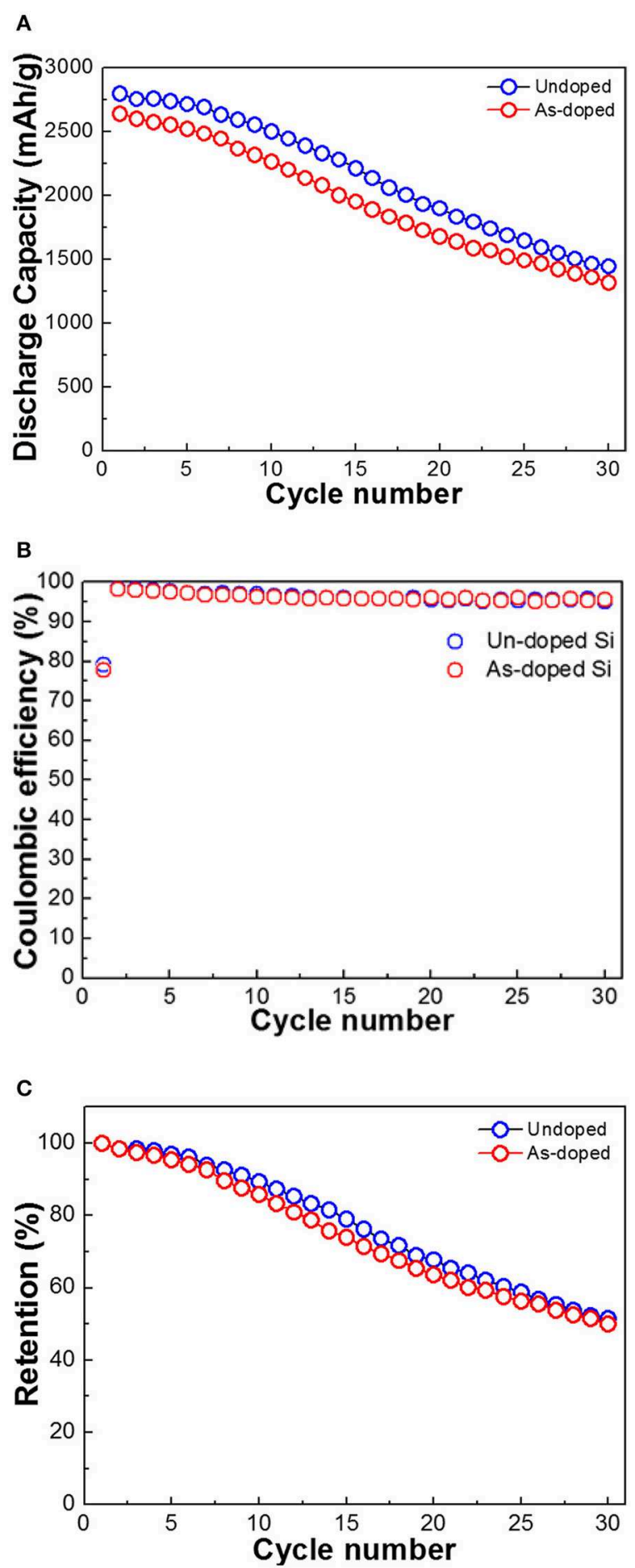

FIGURE 4 | Electrochemical performance of undoped and As-doped Si anodes: (A) cycle performance, (B) Coulombic efficiency, and (C) capacity retention of negative electrodes vs. $\mathrm{Li}_{/} \mathrm{Li}^{+}$at $1 \mathrm{~A} \mathrm{~g}^{-1}$.

single crystalline $\mathrm{Si}$. It is found that all the peak positions of XRD patterns can be indexed to those of cubic Si. It is interesting to note that the peak positions of As-doped Si anodes were shifted to the lower angles, which is attributed to the increased lattice 


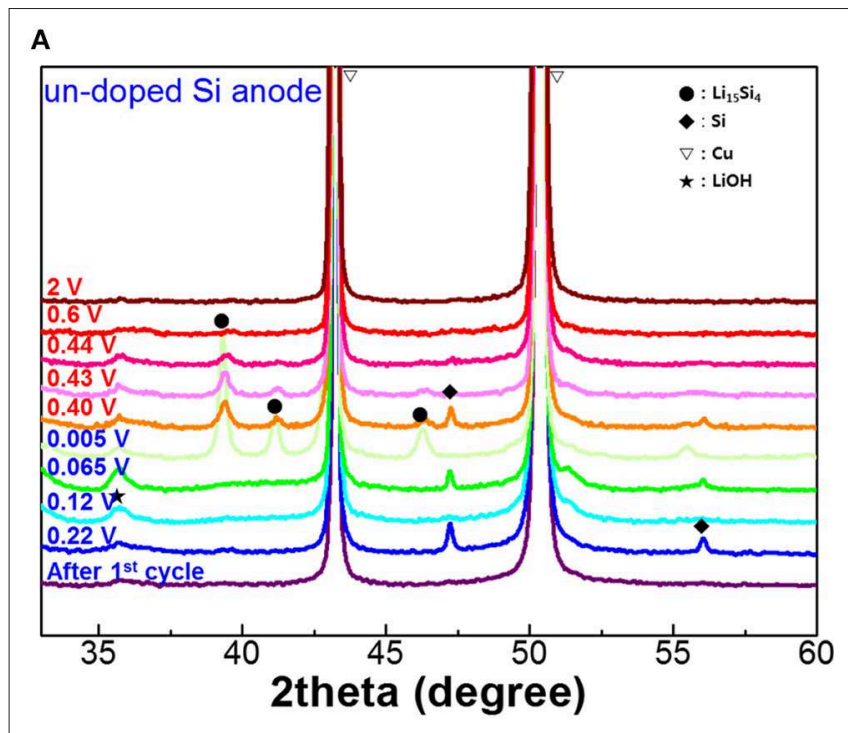

B

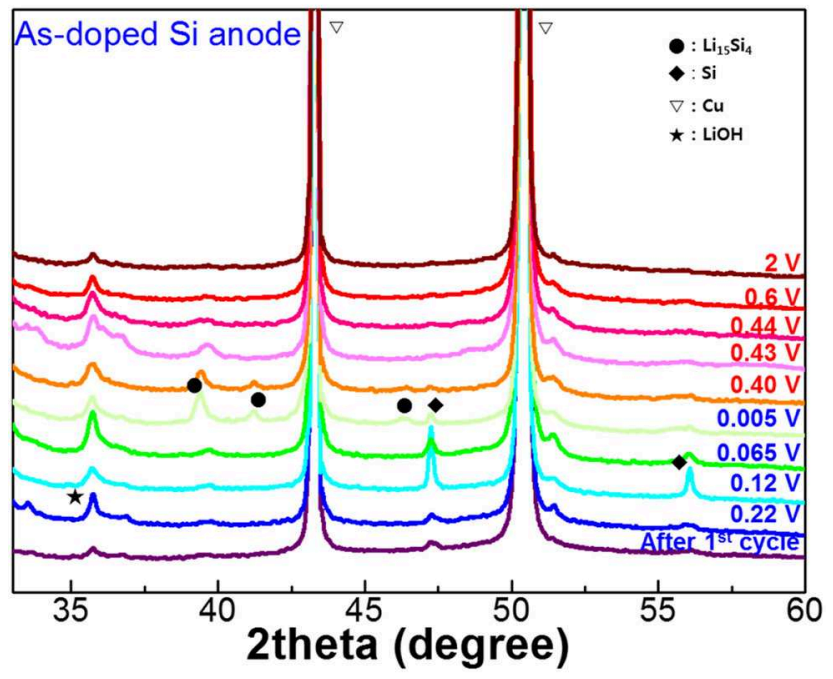

FIGURE 5 | XRD patterns of (A) undoped and (B) As-doped Si anodes after the first lithiation/delithiation as a function of discharge/charge cut-off voltages. The red letters represent discharge voltages, and the blue ones charge voltages.

constant by As-doping into Si structure as depicted in the inset of Figure 2A. Typical stress profiles of undoped and As-doped Si negative electrodes were studied by micro-Raman spectroscopy measurements at room temperature, as shown in Figure 2B. It is well-known that $\mathrm{F}_{2 \mathrm{~g}}$-phonon modes in crystalline $\mathrm{Si}$ can be identified with peaks at around $520 \mathrm{~cm}^{-1}$ (Shimizu et al., 2015). We can easily notice that the peak position for the As-doped $\mathrm{Si}$ anode has been red-shifted, indicating the residual tensile stress of approximately $\sim 1.7 \mathrm{GPa}$ compared to the undoped one. The residual stress can be calculated using the following equation (Wu et al., 2007):

$$
\sigma(\mathrm{MPa})=434 \times \Delta \omega
$$
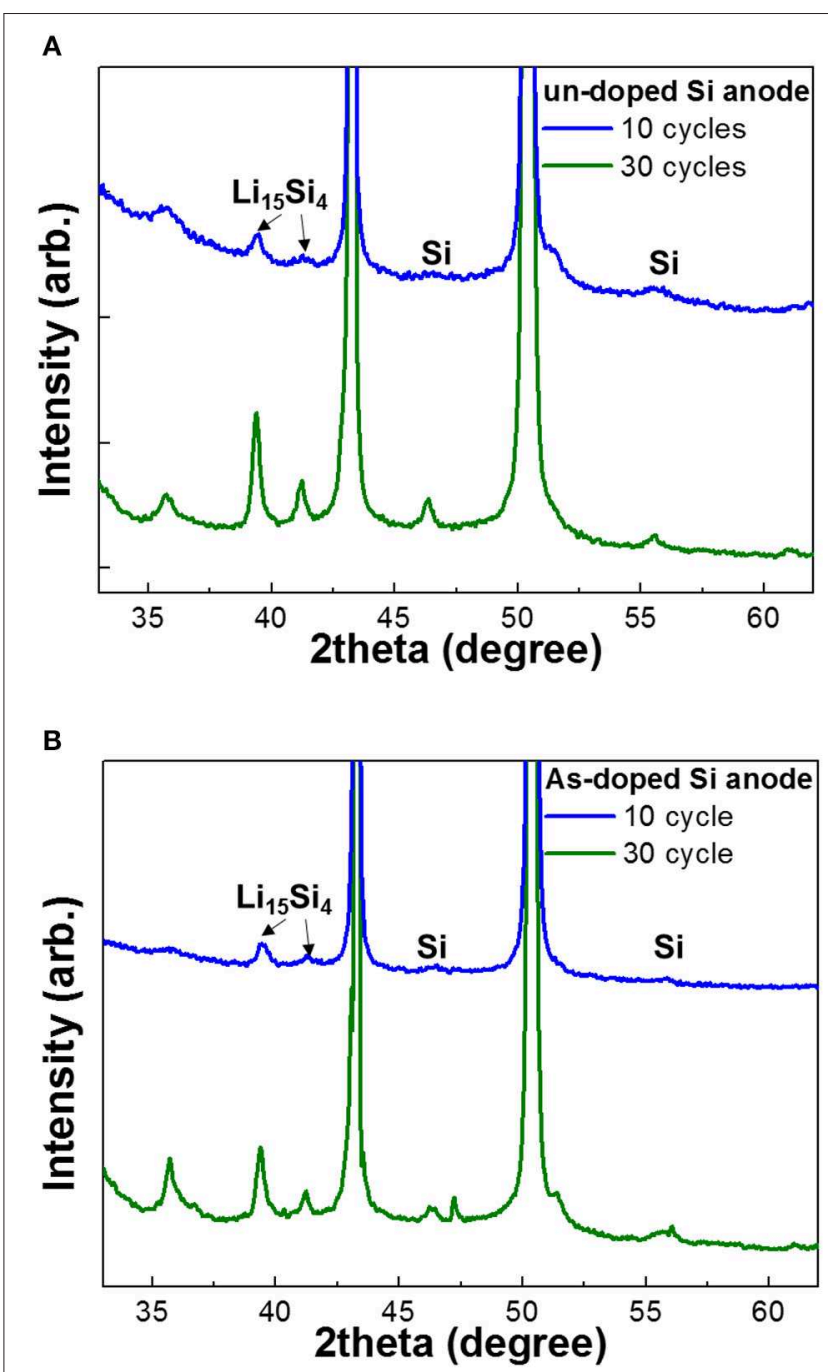

FIGURE 6 | XRD patterns of (A) undoped and (B) As-doped Si anodes vs. cycles.

where $\sigma$ is the biaxial stress, $\Delta \omega$ is the $F_{2 g}$ phonon peak shift. These data are in good agreement with the XRD results in Figure 2A.

In order to evaluate the electrochemical characteristics of $\mathrm{Si}$ negative electrodes with and without dopants, the various electrochemical examinations were performed. Figure 3 illustrates the first charge and discharge behavior of undoped and As-doped Si thick film anodes. It is clearly seen that there are plateaus in the curves, which is attributed to lithiation and delithiation by the reactions of $\mathrm{Si}$ atoms with $\mathrm{Li}$ ions (Chevrier et al., 2010). The initial charge potential plateau is located near $0.1 \mathrm{~V}$, which is in a good agreement with the formation of $\mathrm{Li}_{\mathrm{X}} \mathrm{Si}$ via lithiation of crystallized Si (Han et al., 2016). The first charge and discharge capacity for undoped and As-doped Si negative electrodes are 3,733, 2,782, 3,578, and 2,722 $\mathrm{mAh} \mathrm{g}^{-1}$, respectively. These values correspond to the initial CE of 74.5 and $76.1 \%$, respectively. 

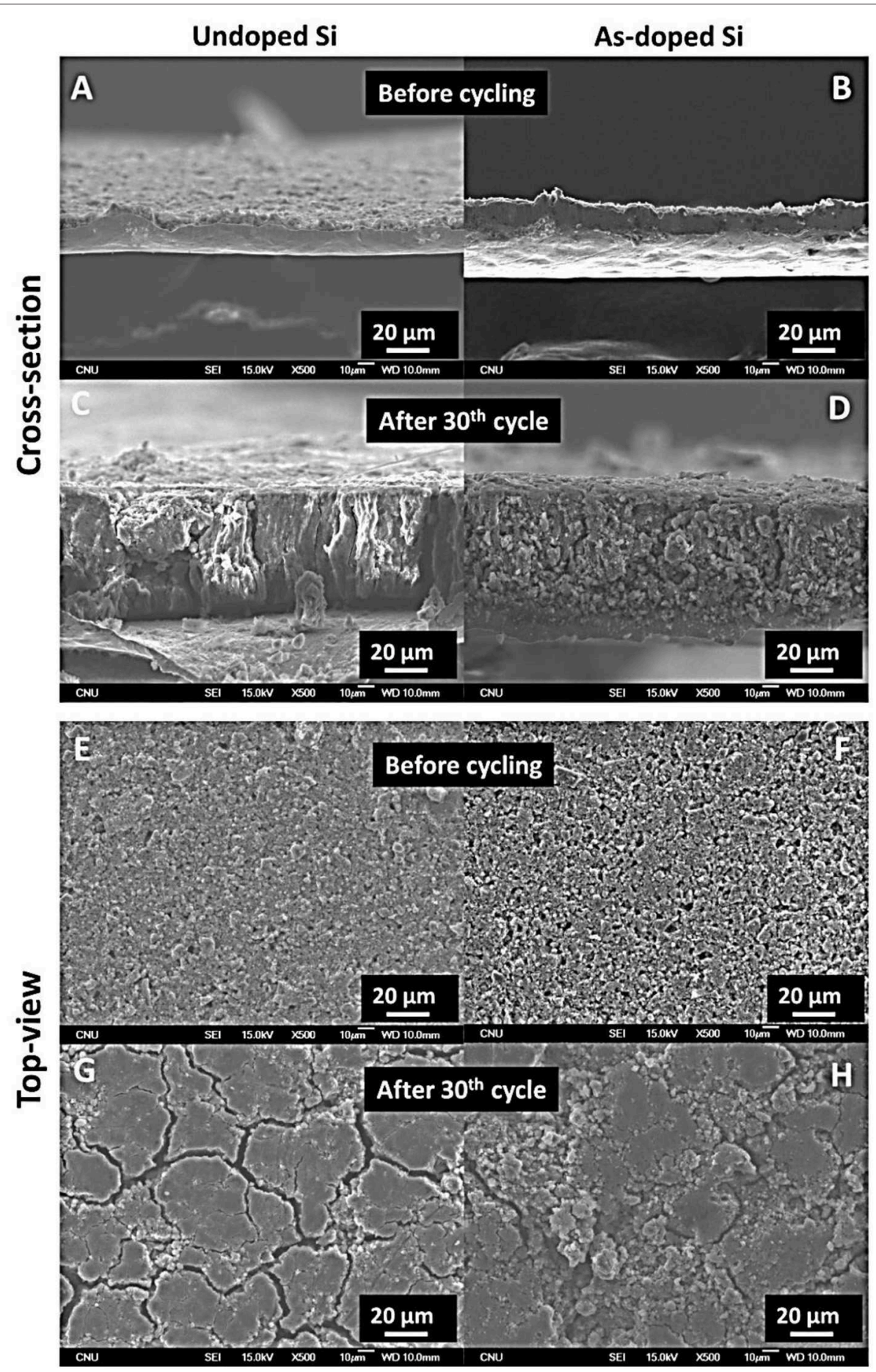

FIGURE 7 | Cross-section SEM micrographs of undoped and As-doped Si anodes (A,B) before and (C,D) after 30th cycle. Top-view SEM micrographs of undoped and As-doped Si anodes $\mathbf{( E , F ) ~ b e f o r e ~ a n d ~} \mathbf{( G , H )}$ after 30th cycle.

Figure 4A illustrates the cycle performance of the cells with the undoped and As-doped Si negative electrodes. The discharge capacities of the LIBs with both types of the anodes were evaluated in a cut-off potential range of $0.005-2.000 \mathrm{~V}$ as seen in Figure 3. The discharge capacity of LIB with undoped $\mathrm{Si}$ negative electrode was higher than that of As-doped Si in all cycle ranges. One can easily observe that the discharge capacity fading of undoped Si anode is comparable with that of As-doped Si. Notably, a fading rate of discharge capacity with the undoped Si negative anode changed more sharply near 10 cycles. The 


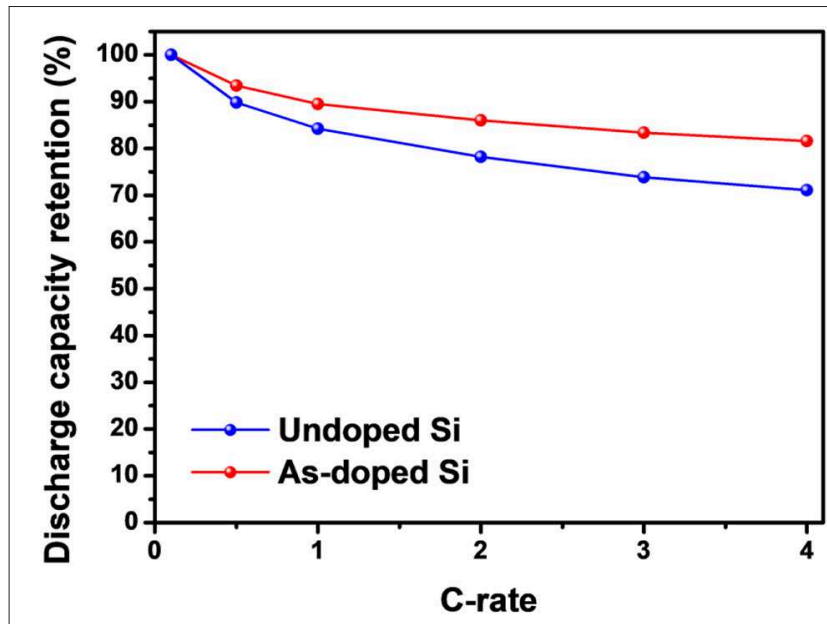

FIGURE 8 | Rate capability of undoped and As-doped Si electrodes from 0.1 to $4.0 \mathrm{C}(1 \mathrm{C}=1.0 \mathrm{~A} / \mathrm{g})$.

initial discharge capacities of both anodes were decreased by approximately 50\% after 30 cycles and their discharge capacities arrived at similar extent. Figure $4 \mathbf{B}$ shows the $\mathrm{CE}$ of both investigated materials. The subsequent cycle $\mathrm{CE}$ of cells with undoped and As-doped Si anodes start from 98.5 and $99.6 \%$ and further reaches over 95.0 and $95.8 \%$ after 30 cycles, respectively. In addition, the capacity retentions of both cells were measured as shown in Figure 4C. Despite the discharge capacity of undoped $\mathrm{Si}$ anode was higher than that with As-doped Si one, its capacity retention displays similar trends, corresponding to about $50 \%$ of retention. This phenomena can be explained by the fact that the As-doped $\mathrm{Si}$ anodes consumed more Li-ions for the generation of a solid-electrolyte interface (SEI) compared with the undoped Si anode and high energy barrier in energy band diagram, which will be mentioned later.

Figure 5 depicts 2theta X-ray rocking curves of the undoped and As-doped Si negative electrodes with various cut-off voltages (OCV) in charging/discharging states after the 1st cycle. After the formation process, there is no sign of the $\mathrm{Li}_{15} \mathrm{Si}_{4}$ phase presence in XRD curves for undoped and As-doped Si anodes. Besides, XRD patterns of both undoped and As-doped Si anodes discharged up to a potential of $0.005 \mathrm{~V}$ clearly revealed the presence of the peaks at $39^{\circ}$ and $41^{\circ}$, indicating the formation of $\mathrm{Li}_{15} \mathrm{Si}_{4}$ silicate ( $\mathrm{Li}$ and Dahn, 2007). It is essential to note that the intensity of XRD patterns of undoped Si negative electrodes was much higher than that of As-doped Si one. This implies that the phase transition of undoped $\mathrm{Si}$ anodes into $\mathrm{Li}_{15} \mathrm{Si}_{4}$ phase is easier to occur compared to the doped electrode. In the discharging states, the peak intensity of $\mathrm{Li}_{15} \mathrm{Si}_{4}$ reduced steadily with increasing the OCV, and finally disappeared at OCV of $2 \mathrm{~V}$ for undoped and As-doped Si anodes.

In order to estimate the phase transition of undoped and As-doped Si anodes, we performed XRD analysis as a function of cycle number as illustrated in Figure 6. No Li silicide peaks were found in the XRD spectra of the samples before cycling (not shown in this paper). The $\mathrm{Li}_{15} \mathrm{Si}_{4}$ phase peaks in XRD
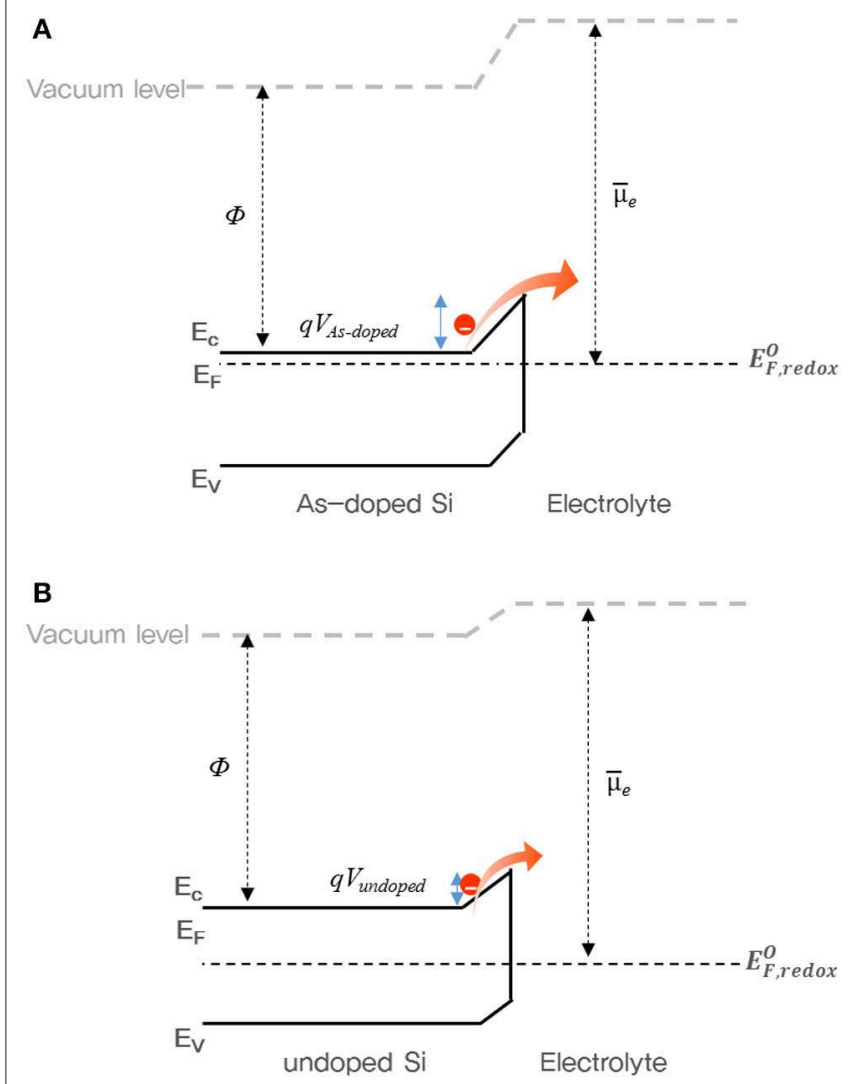

FIGURE 9 | Energy band diagram of cells with (A) As- and (B) undoped Si negative electrodes during charging state. $E_{C}, E_{V}, E_{F}, E_{F, r e d o x}$, and $u_{e}$ indicate the conduction band edge, valence band edge, Fermi level, redox fermi level of electrolyte, and electrochemical potential of the electrolyte.

curves after 10 and 30 cycles were easily confirmed as seen in Figures $6 \mathbf{A}, \mathbf{B}$. Indeed, the XRD intensity of $\mathrm{Li}_{15} \mathrm{Si}_{4}$ phase in undoped $\mathrm{Si}$ anode is much higher than that in As-doped Si, which is consistent with the results obtained in Figure 5. In addition, the gradual increase of $\mathrm{Li}_{15} \mathrm{Si}_{4}$ phase upon 30 cycles is assigned to the activation process of Li-ion pathway in the electrode-electrolyte system, despite the fact that activation was accompanied by the fast capacity fading of both electrodes as observed in galvanostatic cycling.

Furthermore, we investigated the morphology of the electrodes at their cross-section and surface before and after 30 cycles using SEM imaging. Cross-sectional SEM images of undoped and As-doped Si (Figures 7A,B) showed that both electrodes have the thickness of approximately $20 \mu \mathrm{m}$ before cycling, while after 30 cycles, thickness increased drastically $(>200 \%)$ that can be observed in Figures 7C,D. Besides, both samples exhibited similar changes in volume expansion. From the top-view images of undoped Si vs. Asdoped Si (Figures 7E-H), one can see that the morphology of electrodes looked the same before and after cycling. The extensive formation of cracks and partial pulverization of active electrode material after 30 cycles was observed in both cases shown in Figures 7G,H. Accordingly, SEM results 
supported the capacity fading phenomena revealed by the galvanostatic tests earlier for the undoped and As-doped Si anodes.

Figure 8 compares the discharge capacity retentions of undoped and As-doped $\mathrm{Si}$ electrodes at various current densities from 0.1 to $4.0 \mathrm{C}(1 \mathrm{C}=1.0 \mathrm{~A} / \mathrm{g})$, respectively. Here, undoped Si exhibits considerable fading in discharge capacity at higher current densities compare with As-doped Si. More specifically, at the highest current density of $4.0 \mathrm{C}$, the undoped Sibased electrode shows $69 \%$ in capacity retention vs. 0.1 C. By contrast, the As-doped Si demonstrates better rate performance with $\sim 80 \%$ capacity retention. Obvious improvement in rate capability can be ascribed to the As-doping effect in $\mathrm{Si}$ structure and will be further discussed with the electronic energy band diagram.

To understand the charge/discharge characteristics of undoped and As-doped Si negative electrodes, we took the electronic energy band diagram of both $\mathrm{Si}$ anode-electrolyte interfaces into consideration as illustrated in Figure 9. It is well-known that when the semiconductor-electrolyte interface is formed, electrolyte and semiconductor reach electrochemical equilibrium by matching their electrochemical potentials. This occurs by charge transfer between both media (Prados et al., 2014). Considering this, each barrier heights of undoped and Asdoped $\mathrm{Si}$ anodes are represented in Figures 9A,B, respectively. It is noteworthy to mention that the energy barrier height from

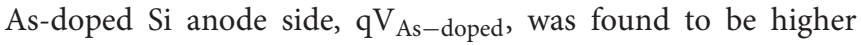
than $\mathrm{qV}_{\text {undoped }}$ of undoped $\mathrm{Si}$ anode after equilibrium state. Hence, the flux of electrons across the electrolyte in As-doped Si with the increased potential barrier height can be impeded, thus hindering charge flow in the cell. In previously published works, the addition of dopants such as $\mathrm{B}$ and $\mathrm{P}$ decreased the lattice spacing of $\mathrm{Si}$ anodes and led to the increase of the insertion energy of Li ions, thus suppressing the discharging and charging capacity in LIBs (Long et al., 2011; Domi et al., 2016). However, the charging/discharging capacities of As-doped Si anodes have a different aspect, apart from volume change. To understand the electrochemical behaviors of cells with As-doped Si anodes, the energy band diagrams were illustrated, as shownv in Figure 9. It was found that the potential barrier of cells with As-dopant is much higher than that of undoped Si anode. We can infer that the higher potential barrier on the anode side by highly n-type doping results in the reduced charging/discharging capacity of As-doped Si anodes, compared to that of undoped $\mathrm{Si}$ ones. This is in a fair agreement with the results of Figures 5, 6. Moreover, we consider that slightly higher CE of As-doped Si

\section{REFERENCES}

Chevrier, V., Zwanziger, J., and Dahn, J. (2010). First principles study of Li-Si crystalline phases: charge transfer. Electronic structure, and lattice vibrations. J. Alloys Compounds 496, 25-36. doi: 10.1016/j.jallcom.2010.01.142

Domi, Y., Usui, H., Shimizu, M., Kakimoto, Y., and Sakaguchi, H. (2016). Effect of phosphorus-doping on electrochemical performance of silicon negative electrodes in lithium-ion batteries. ACS Appl. Mater. Interfaces 8, 7125-7132. doi: 10.1021 /acsami.6b00386 negative electrode is related to the potential barrier. A lower charge/discharge capacity leads to the suppression of the phase transition from $\mathrm{c}-\mathrm{Si}$ to a-Si, which may result in the improved $\mathrm{CE}$, corresponding to the XRD results.

\section{CONCLUSION}

In summary, the electrochemical performance of the cells with As-doped $\mathrm{Si}$ negative electrodes was investigated. The ballmilling of single-crystalline Si wafers was used to fabricate the anodes, which particles were irregular and agglomerated microflakes. The XRD and Raman analysis revealed that As-doped $\mathrm{Si}$ anodes have larger lattice spacing compared to undoped $\mathrm{Si}$ samples, indicating the residual tensile stress of approximately $\sim 1.7 \mathrm{GPa}$. Despite the increased lattice spacing in As-doped Si anode, its charge/discharge capacity was lower compared with that of undoped $\mathrm{Si}$ anodes. The Coulombic efficiency and cyclic retention of As-doped Si negative electrodes, however, were comparable to those of the undoped $\mathrm{Si}$ anode. This effect was attributed to the increased potential barrier height on the side of As-doped $\mathrm{Si}$, affecting the electrochemical performance of LIBs with the As-doped anode. We believe that this study will provide an effective approach to achieve the enhanced electrochemical performances of LIBs with Si-based anode derived from electronic waste materials.

\section{DATA AVAILABILITY}

The datasets generated for this study are available on request to the corresponding author.

\section{AUTHOR CONTRIBUTIONS}

ML and DY conceived design of the study and carried out the experiment; UL, ZB, and S-SK contributed to the interpretation of the results; NU, AM, $\mathrm{ZB}$, and S-SK wrote sections of the manuscript. All authors contributed to manuscript revision, read and approved the submitted version.

\section{ACKNOWLEDGMENTS}

The authors acknowledge the financial support of the KETEP (Grant No.20164010201070) and the Nazarbayev University by the research grant No.110119FD4504 Development of 3D all solid-state thin film Li-ion microbatteries. 
Kim, H., Chou, C.-Y., Ekerdt, J. G., and Hwang, G. S. (2011). Structure and properties of Li- Si alloys: a first-principles study. J. Phys. Chem. C. 115, 2514-2521. doi: 10.1021/jp1083899

Ko, M., Chae, S., and Cho, J. (2015). Challenges in accommodating volume change of Si anodes for Li-ion batteries. Chem. Electro. Chem. 2, 1645-1651. doi: 10.1002/celc.201500254

Legrain, F., and Manzhos, S. (2015). Aluminum doping improves the energetics of lithium. sodium, and magnesium storage in silicon: A first-principles study. J. Power Sourc. 274, 65-70. doi: 10.1016/j.jpowsour.2014.10.037

Li, J., and Dahn, J. (2007). An in situ X-ray diffraction study of the reaction of Li with crystalline Si. J. Electrochem. Soc. 154, A156-A161. doi: 10.1149/1.2409862

Liu, X. H., Zhong, L., Huang, S., Mao, S. X., Zhu, T., and Huang, J. Y. (2012). Size-dependent fracture of silicon nanoparticles during lithiation. ACS Nano. 6, 1522-1531. doi: 10.1021/nn204476h

Long, B. R., Chan, M. K., Greeley, J. P., and Gewirth, A. A. (2011). Dopant modulated Li insertion in Si for battery anodes: theory and experiment. J Phys Chem C. 115, 18916-18921. doi: 10.1021/jp2060602

Ma, D., Cao, Z., and Hu, A. (2014). Si-based anode materials for Li-ion batteries: a mini review. Nano Micro Lett. 6, 347-358. doi: 10.1007/s40820-014-0008-2

McSweeney, W., Lotty, O., Glynn, C., Geaney, H., Holmes, J. D., and O’Dwyer, C. (2014). The influence of carrier density and doping type on lithium insertion and extraction processes at silicon surfaces. Electrochim. Acta 135, 356-367. doi: 10.1016/j.electacta.2014.05.035

Mukanova, A., Jetybayeva, A., Myung, S. -T., Kim, S., and Bakenov, Z. (2018b). A mini-review on the development of Si-based thin film anodes for Li-ion batteries. Mater Today Energy 9, 49-66. doi: 10.1016/j.mtener.2018.05.004

Mukanova, A., Nurpeissova, A., Kim, S. -S., and Myronov, M., Bakenov, Z. (2018a). N-type doped silicon thin film on porous $\mathrm{Cu}$ current collector as a negative electrode for Li-ion batteries. ChemistryOpen. 7, 92-96 doi: 10.1002/open.201700162

Nitta, N., Wu, F., Lee, J. T., and Yushin, G. (2015). Li-ion battery materials: present and future. Mater Today 18, 252-264. doi: 10.1016/j.mattod.2014.10.040

Peng, B., Cheng, F., Tao, Z., and Chen, J. (2010). Lithium transport at silicon thin film: barrier for high-rate capability anode. J. Chem. Phys. 133:034701. doi: $10.1063 / 1.3462998$

Prados, A., Ranchal, R., and Pérez, L. (2014). Blocking effect in the electrodeposition of Bi on $\mathrm{n}-\mathrm{GaAs}$ in acidic electrolytes. Electrochim. Acta 143, 23-28. doi: 10.1016/j.electacta.2014.07.137

Rousselot, S., Gauthier, M., Mazouzi, D., Lestriez, B., Guyomard, D., and Roué, L. (2012). Synthesis of boron-doped Si particles by ball milling and application in Li-ion batteries. J. Power Sources. 202, 262-268. doi: 10.1016/j.jpowsour.2011.11.045

Ryu, J. H., Kim, J. W., and Sung, Y. -E., Oh, S. M. (2004). Failure modes of silicon powder negative electrode in lithium secondary batteries. Electrochem Solid State Lett. 7, A306-A309. doi: 10.1149/1.1792242

Shi, F., Song, Z., Ross, P. N., Somorjai, G. A., Ritchie, R. O., and Komvopoulos, K. (2016). Failure mechanisms of single-crystal silicon electrodes in lithium-ion batteries. Nat. Commun. 7:11886. doi: 10.1038/ncomms11886
Shimizu, M., Usui, H., Suzumura, T., and Sakaguchi, H. (2015). Analysis of the deterioration mechanism of Si electrode as a Li-ion battery anode using Raman microspectroscopy. J. Phys. Chem. C. 119, 2975-2982. doi: 10.1021/jp5121965

Talla, G., Guduru, R. K., Li, B. Q., and Mohanty, P. S. (2015). Doped-SiAg composite electrodes for Li-ion batteries. Solid State Ionics 269, 8-13. doi: 10.1016/j.ssi.2014.09.021

Umirov, N., Seo, D. H., Jung, K. N., Kim, H. Y., and Kim, S. S. (2019b). Ni added Si-Al Alloys with Enhanced $\mathrm{Li}^{+}$Storage Performance for Lithium-Ion Batteries. J. Electrochem. Sci. Technol. 10, 82-88. doi: 10.5229/JECST.2019.10. 1.82

Umirov, N., Seo, D. H., Kim, T., Kim, H. Y., and Kim, S. S. (2019a). Microstructure and electrochemical properties of rapidly solidified $\mathrm{Si}-\mathrm{Ni}$ alloys as anode for lithium-ion batteries. J. Industr. Eng. Chem. 71, 351-360. doi: 10.1016/j.jiec.2018.11.046

Wang, B., Li, X., Zhang, X., Luo, B., Zhang, Y., and Zhi, L. (2013). Contactengineered and void-involved silicon/carbon nanohybrids as lithium-ionbattery anodes. Adv Mater. 25, 3560-3565. doi: 10.1002/adma.201300844

Wang, J., Meng, X., Fan, X., Zhang, W., Zhang, H., and Wang, C. (2015). Scalable synthesis of defect abundant Si nanorods for high-performance Li-ion battery anodes. ACS nano. 9, 6576-6586. doi: 10.1021/acsnano.5b02565

Wang, Y., and Dahn, J. (2006). Comparison of the reaction of Li x Si or Li0. 81C6 with 1 M LiPF6 EC: DEC electrolyte at high temperature. Electrochem Solid State Lett. 9:A340-3. doi: 10.1149/1.2200137

Wen, Z., and Tian, F. (2013). Cu-doped silicon film as anode for lithium ion batteries prepared by ion-beam sputtering. Int. J. Electrochem. Sci. 8, 10129-10137.

Wu, X., Yu, J., Ren, T., and Liu, L. (2007). Micro-Raman spectroscopy measurement of stress in silicon. Microelectr. J. 38, 87-90. doi: 10.1016/j.mejo.2006.09.007

Yao, Y., McDowell, M. T., Ryu, I., Wu, H., Liu, N., Hu, L., et al. (2011). Interconnected silicon hollow nanospheres for lithium-ion battery anodes with long cycle life. Nano Lett. 11, 2949-2954. doi: 10.1021/nl201470j

Yi, R., Zai, J., Dai, F., Gordin, M. L., and Wang, D. (2013). Improved rate capability of $\mathrm{Si}-\mathrm{C}$ composite anodes by boron doping for lithiumion batteries. Electrochem. commun. 36, 29-32. doi: 10.1016/j.elecom.2013. 09.004

Conflict of Interest Statement: The authors declare that the research was conducted in the absence of any commercial or financial relationships that could be construed as a potential conflict of interest.

Copyright (C) 2019 Lee, Yoon, Lee, Umirov, Mukanova, Bakenov and Kim. This is an open-access article distributed under the terms of the Creative Commons Attribution License (CC BY). The use, distribution or reproduction in other forums is permitted, provided the original author(s) and the copyright owner(s) are credited and that the original publication in this journal is cited, in accordance with accepted academic practice. No use, distribution or reproduction is permitted which does not comply with these terms. 